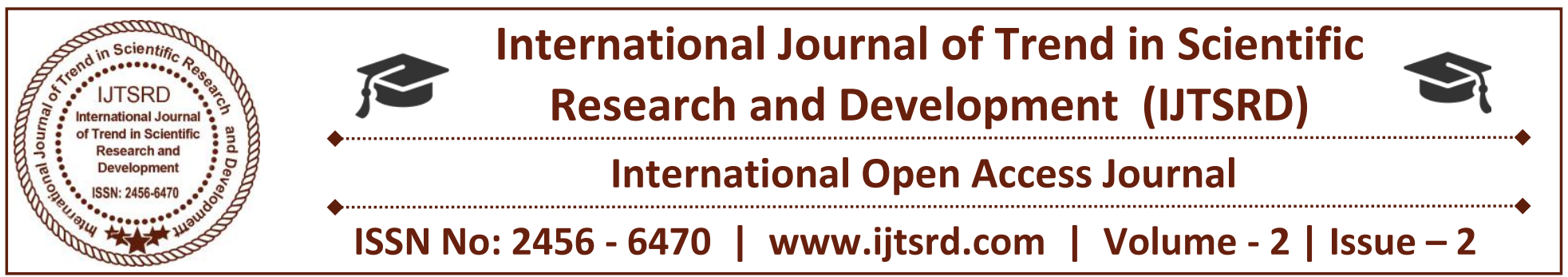

\title{
Conceptualization of Management of Healthcare Services
}

\author{
Mansour Moklif Alanazi, MD \\ Family and Geriatric Medicine, Pain \\ Management Consultant; \\ Director of Family Medicine in King Fahd \\ Military Medical Complex, Saudi Arabia
}

\author{
Mohammed Ratoubi Alanazi, PhD \\ Chief of Curriculum Development in \\ Military School of Allied Health Sciences \\ in Saudi National Guard Health Affairs; \\ Assistant Professor in King Saud bin \\ Abdulaziz University for Health \\ Sciences, Saudi Arabia
}

\section{ABSTRACT}

Healthcare services are provided in a dynamic and complex system. Managing healthcare organization require from healthcare managers to determine the need for and scope of change and to manage the transition process to a sustainable result. It is very important to understand the organizational structure in healthcare system because there are multi relationships between different groups of people who working in healthcare organizations. Healthcare organizational performance assess to ensure that healthcare services provided in high quality, high productivity, safety and cost effectiveness manner. Conceptualize the management of healthcare services provide a holistic picture and help to improve quality of healthcare services.

Keywords: Management, Healthcare, Health system, Organizational structure, Performance, Organizational theory, Conceptualization

\section{Introduction}

Healthcare services are provided in a dynamic and complex system. Various daily process, activities and functions that happen in healthcare organizations, in addition, to rapidly changes increase complexity of healthcare system. This dynamic and complexity increase the possibility of risk in healthcare system. Therefore, patients encounter to medical errors in health system in such frightful numbers who injures, disable and/or die due to those errors. It is necessary to conceptualize management of healthcare services to understand how healthcare managers should take their decision in such dynamic and complex system and how performance of healthcare organization should be grow into culture of patient safety and reduce medical errors.

\section{Characteristics of Healthcare System}

Healthcare services exist in changeable environment. Managing healthcare organization require from healthcare managers to determine the need for and scope of change and to manage the transition process to a sustainable result. Concept of change means ' a movement from one state of being or understanding to another' (Lioyd, Johnstone et al. 2004). Healthcare organizations have four levels of drivers change which are individual, the work team, the organization and the system or environment (Lioyd, Johnstone et al. 2004: 147). Healthcare managers need to analyze dimensions of change in health system to end up with safe health system and avoid risks to patients. There are many techniques to analyze dimensions of change. Lioyd et al (2004) list the following techniques are:

- SWOT technique which is: Strengths, Weakness, Opportunities and Threats.

- Critical Incident Review.

- External consultant review of organizational performance.

Furthermore, to above techniques; there what called "7S" checklist that according to Waterman et al (1980) when all seven applied in an organization, it 
will achieve its objectives in efficient way. The seven factors are strategy, structure, system, style, staff, skills and shared values and beliefs (Lioyd, Johnstone et al. 2004: 155).

Conceptualize work design in healthcare sector is important to enhance patient safety and avoid possible risk inside healthcare organizations. It is very important to know who works in healthcare, types of job design activities and characteristics of the health workforce to discover why errors happen in healthcare system. In addition, it is crucial to recognize that healthcare systems have very wide professional specialties or categories. The numbers of professional categories expand from 12 specialties in 1950 to 105 in 1980 (Courtney, Klinken et al. 2002). There are many types of job design activities, but the most common designs are job rotation, job enlargement, job enrichment, flexible working hours, job sharing and multi-skilling (Courtney, Klinken et al. 2002: 233-238). Health workforce has unique characteristics, for example, in most countries nurses are female and there are diverse culture and language between patients and healthcare professionals. Therefore, conceptualize work design in healthcare institutions is essential to put strategic plans to minimize risk and improve patient safety.

\section{Organizational Structure}

Organizational structure and performance had been emphasized from a long of time to increase productivity and quality. There are a lot of theorists who focus on organizational structure and performance, such as, Frederick W.Taylor (1911), Henri Fayol (1919), LyndallUrwick (1944), Luther Gulick (1937) and Max Weber (1900s) (Courtney, Nash et al. 2002: 265). It is very important to understand the organizational structure in healthcare system because there are multi relationships between different groups of people who working in healthcare organizations. There are relationship between patients and health workers from one side and relationship between health workers themselves in the other side. Management scientists focus on management practices to clarify division of labor, chain of command, rules and regulation and coordination of activities to provide services to customers in costeffective and efficient way (Courtney, Nash et al. 2002). Focus on management practices in healthcare organizations is important to create safer health system. For example, if chain of command not clear nurse could confuse and make mistakes if different and clash orders came from doctor and head nurse for the same process.

There are different types of organizational structure. The most common types in healthcare organizations are functional, divisional, product/market and matrix designs (Courtney, Nash et al. 2002). The functional organizational design is grouping workers who doing the same functions or tasks together. In healthcare organizations, medical division, nurse division, allied health division, pharmacy division and administrative services are examples of functional organizational structure. The divisional and product/market designs used in a broader product range which functional structure cannot did. An example of this design in healthcare system are division of surgery which could contain large product range, such as, vascular, cardiac, ENT, head and neck, neurosurgery and anesthetics. The matrix design contains characteristics of both functional and divisional designs. An example of matrix design in healthcare is hospitals, where 'continuous interchange between product and the function' occurs (Griffith 1995 cited in Courtney, Nash et al. 2002). Matrix design play vital role to improve patient safety and reduce medical errors because enhance cooperation and communication between members of multidisciplinary departments are features of this design.

\section{Organizational Performance}

Measuring organizational performance in healthcare system is required to conceptualize management of healthcare services. Healthcare managers should assess performance of their healthcare organizations to ensure that the mission and the goals of their organizations are achieved. Therefore, it is very difficult to create safety culture while punitive approach exists. According to Isouard et al (2002) improve patient care performance require efficacious, appropriate, timely, effective, well coordinated, safe, efficient, and caring and respectful care (Isouard, Messum et al. 2002: 336). There are many approaches to improve health organizational performance. The main approaches include total quality management, best practice, benchmarking, balanced scorecard, risk management and evidence -based decision making. Improving health organizational performance affects quality of care and patient safety. Therefore, it is essential to measure health care organizational 
performance to ensure safety of patients and avoid adverse events.

Healthcare organizational performance assess to ensure that healthcare services provided in high quality, high productivity, safety and cost effectiveness manner. Performance of healthcare organizations evaluate in terms of efficacy, effectiveness, appropriateness, productivity and efficiency (Flood, Shortell et al. 1997). There are some factors that should be considered in evaluation of organization performance. Knowing nature of organization, the impact of time frames, the domains of the activities being evaluated, the level of analysis and the perspective of interested stakeholders in organization are the most factors that should be realized in assessing organization performance (Flood, Shortell et al. 1997: 392).

\section{Organizational Theory}

Organization theory is important to be understood in healthcare organizations. Organization theory is defined as ' the systemic study and careful application of knowledge about how people - as individuals and as groups- act within organization' (Organizational Theory 2010). Healthcare organizations as any organization consist of group of people (doctors, nurses, technicians, pharmacists, and administrators) who work together for specific purposes. Harm patients in healthcare organizations is certainly not any of these purposes, however, it is happen. Therefore, understanding organizational theory and how people in health organizations act either with patients or between themselves can help in improve patient safety and avoid preventable adverse events.

Healthcare organizations are unique. There are special characteristics that signalize or differentiate healthcare organizations than other types of organizations. According to Shortell and Kaluzny (2000:13) the most differences are:

1. Defining and measuring output are more difficult

2. The work involved is more variable and complex

3. More of the work is of an emergency and nondeferrable nature

4. The work permits little tolerance for ambiguity or error

5. The work activities are highly interdependent, requiring a high degree of coordination among diverse professional groups
6. Dual lines of authority which create problems of coordination and accountability and confusion of roles.

There are several perspectives that show how organization works. Organization theory helps to draw a ' mental map' that clarifies how things connected and how things happen. The major perspectives of how organizations work include classical bureaucratic theory, the scientific management school, the human relations school, contingency theory, resource dependence theory, the strategic management perspective, population ecology theory and institutional theory (Shortell and Kaluzny 2000: 20). The previous theories play major role to demonstrate structure and functioning of healthcare organizations.

\section{Conclusion}

In brief, conceptualize the nature of healthcare organizations allow policy makers to understand why medical errors occur and why health workers make mistakes. It can be clearly seen that conceptualize the management of healthcare services provide a holistic picture regarding the unique characteristics of healthcare systems. The understanding of organizational structure and performance is essential to achieve better and safer healthcare system. Thus, the conceptualization of management of healthcare services will help to improve safety in healthcare organizations.

\section{References}

1. Lloyd, P., Johnstone, P. L., \& Dwyer, J. (2004). Managing and Leading change. In M. Harris \& Associates (Eds.), Managing Health Services: concepts and practice. Marrickville: Southwood Press.

2. Courtney, M., Klinken, A., Majoor, J., \& Ibrahim, J. (2002). Work design in health care In M. Harris \& Associates (Eds.), Managing Health Services: Concepts and Practice. Marrickville: Sothwood Press.

3. Courtney, M., Nash, R., \& Montgomery, J. (2002). Strategy and Orgnizational Design in health care. In M. Harris \& Associates (Eds.), Manging Health Services: Concepts and Practice. Marrickville: Southwood Press.

4. Isouard, G., Messum, D., Hanson, S., McAlpin, S., \& Briggs, D. (2002). Improving organizational 
performance in health care. In M. Harris \& Associates (Eds.), Manging Health Services: concepts and practice. Marrickville: Southwood Press.

5. Flood, A., Shortell, S., \& Scott, W. R. (1997). Organizational Performance: Managing for Efficiency and Effectiveness. In S. Shortell\& A. Kaluzny (Eds.), Essentials of Health care Management. Albany: Delmar Publishers.

6. Organizational Theory. (2010). Organizational Theory Retrieved 13 April 2010, from http://www.scribd.com/doc/29394556/Organizatio nal-Theory

7. Shortell, S., \&Kaluzny, A. (2000). Health Care Managemenet: organization design and behaviour (4th ed.). Albany: Delmar Thomson Learning.

8. Waterman Jr., Robert H., Peters, Thomas J., and Julien R. Phillips. (1980). Structures is not Organization. Business Horizons 23, no. 3: 14

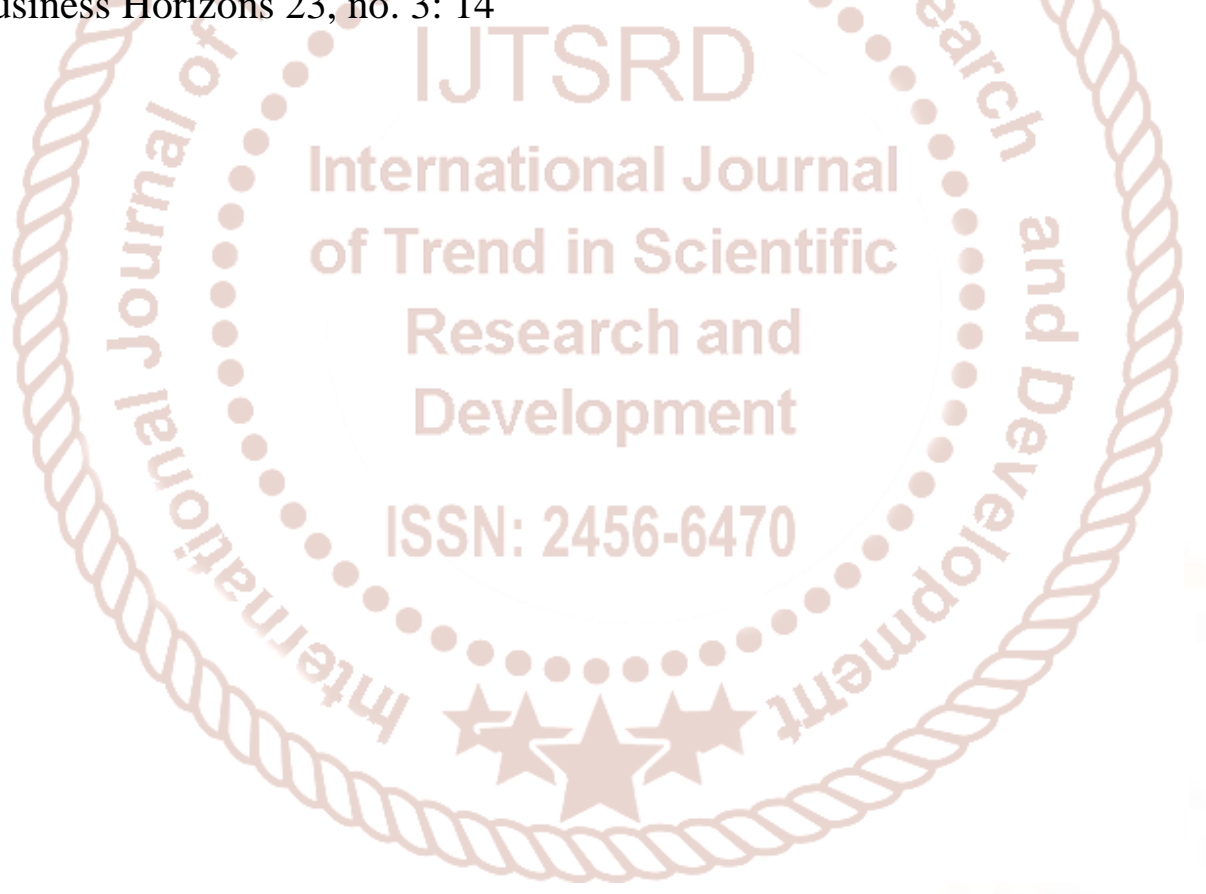

